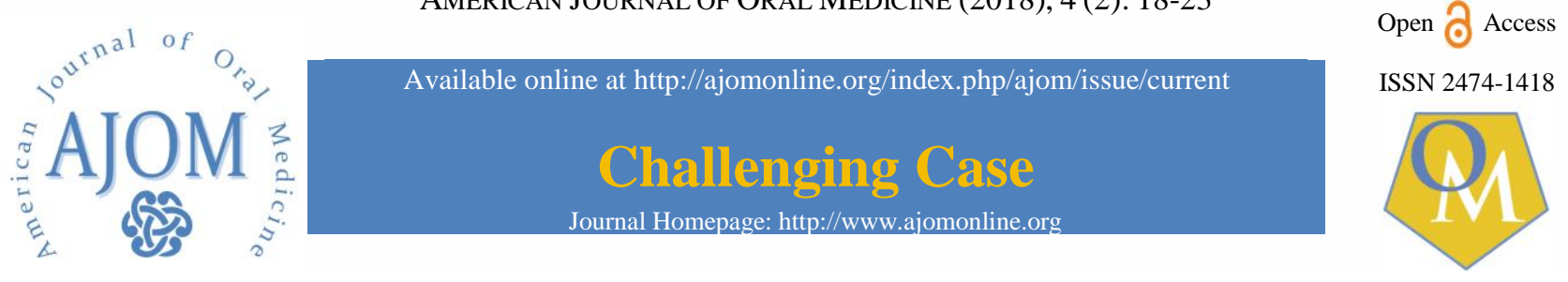

\title{
Plasma cell mucositis: an unusual case
}

\author{
Keeratika Wongtim $^{1}$, Keskanya Subbalekha ${ }^{2}$, Risa Chaisuparat ${ }^{3}$, and Kobkan Thongprasom ${ }^{1}$ \\ 1. Department of Oral Medicine, Faculty of Dentistry, Chulalongkorn University, Bangkok, Thailand \\ 2. Department of Oral and Maxillofacial Surgery, Faculty of Dentistry, Chulalongkorn University, Bangkok, Thailand \\ 3. Department of Oral Pathology, Faculty of Dentistry, Chulalongkorn University, Bangkok, Thailand
}

\author{
PUBLICATION HISTORY \\ Received: 11 May 2018 \\ Returned for revision: 01 June 2018 \\ Received in revised form: 30 June 2018 \\ Accepted: 01 July 2018 \\ Corresponding author: \\ Kobkan Thongprasom \\ Department of Oral Medicine \\ Faculty of Dentistry \\ Chulalongkorn University \\ Bangkok 10330 \\ Thailand \\ Tel: $+66-2-2188942$ \\ fax: +66-2-2188941 \\ E-mail: kobkan.t@chula.ac.th
}

\section{CHALLENGING CASE}

A 36-year-old female Hepatitis B-positive patient was referred to the Oral Medicine Clinic at the Faculty of Dentistry, Chulalongkorn University, Bangkok in May 2017 complaining of severe oral cavity pain for over 5 months. She had a history of onset nonproductive cough since December 2016. She received no relief from Azithromycin, Codeine, or herbal lozenges. She reported that ulcerations developed on her right and left buccal mucosa 3 days after using the lozenges, thus, discontinued. Her physician prescribed prednisolone $10 \mathrm{mg} 3 \mathrm{x} / \mathrm{day}$, which relieved her cough. However, her nocturnal coughing, burning sensation in her mouth, and dry lips persisted.

During follow-up by her physicians in January 2017, she had conjunctivitis, generalized maculopapules on her extremities, dry scaly patches on her lips, multiple small red spots on the tip of the tongue, and ulcerative and erythematous patches on the left and right buccal mucosa that demonstrated pseudohyphae after a $\mathrm{KOH}$ $10 \%$ assay. Her chest radiograph revealed fibronodular infiltration in both upper lobes, complete blood count showed normal values except for a high eosinophil count (21\%), and a $23 \mathrm{~mm} / \mathrm{h}$ erythrocyte sedimentation rate (ESR) (normal 0-20 $\mathrm{mm} / \mathrm{h}$ in women younger than 50 years old).

Stool examination and urinary analysis appeared normal. Her anti-HIV and acid-fast bacteria (AFB) tests were negative. Clindamycin $300 \mathrm{mg}$ was prescribed 3x/day for 7 days, and multivitamin, clotrimazole troche, triamcinolone acetonide $0.1 \%$, and Lidocaine $\mathrm{HCl}$ gel.

In February 2017, the burning sensation in her mouth worsened, with persistent oral lesions and palatal mucosa erosion. She developed discrete ill-defined blanchable erythematous rashes on her palms, soles, thighs, upper extremities, and upper chest.

Additional investigations were performed including buccal mucosa biopsy. Histopathologically, the lesion demonstrated mucosal hyperplasia, no basal vacuolar change, and was nonmalignant. The laboratory results showed a $25 \%$ eosinophil count, 30 $\mathrm{mm} / \mathrm{h}$ ESR, C-reactive protein (CRP) 3.85 (normal less than $10 \mathrm{mg} / \mathrm{L}$ ), antinuclear antibody (ANA) positive with the titer of $1: 320$ (normal = negative) $($ speckled pattern), Anti-SSA/Ro-positive with strong intensity, and Mycoplasma titer 1:80 (normal less than 1:40). The direct immunofluorescence, indirect immunofluorescence, Anti-dsDNA, Anti-La, Anti-Sm, Anti-nRNP, sputum and stool culture, AFB, and G6PD assays were negative. 
A diagnosis of Mycoplasma pneumoniae and differential diagnosis of Mycoplasma-induced rash and mucositis (MIRM), drug eruption, acute lupus erythematosus, Rowell syndrome, paraneoplastic pemphigus, and drug reaction with eosinophilia and systemic symptoms were made by her physicians. Based on clinical findings, cough and Mycoplasma titer 1:80, Mycoplasma infection

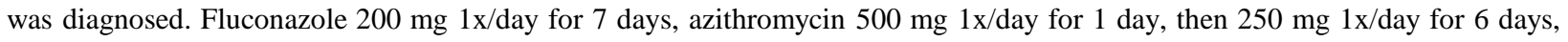
prednisolone $10 \mathrm{mg} \mathrm{2x/day} \mathrm{for} 7$ days, topical steroid, and acetylcysteine were prescribed. However, 2 days later an azithromycin allergy was suspected because of her skin rashes, and levofloxacin $750 \mathrm{mg} \mathrm{1x/day} \mathrm{for} 9$ days was prescribed, replacing azithromycin. One week later, the skin rashes and oral ulcerations improved, however, her palm and arm rashes persisted. Her mycoplasma titer was 1:80, Anti-SSA/Ro-positive with strong intensity, and her eosinophil count and CRP decreased to $0.2 \%$ and less than 0.6 , respectively. Prednisolone treatment was halted, and levofloxacin, fluconazole, and chloroquine were continued. Five days later, although her skin rash and conjunctivitis improved, the oral lesions and burning sensation worsened; thus, prednisolone $5 \mathrm{mg} 3 \mathrm{x} / \mathrm{day}$ was prescribed.

During March-April in 2017, the oral ulcerations on the tongue, buccal mucosa, and hard palate remained. Her chest radiograph was normal. Her physicians treated her with prednisolone $10 \mathrm{mg} \mathrm{1x/day} \mathrm{for} 1$ week, then $5 \mathrm{mg} 1 \mathrm{x} /$ day, and chloroquine $250 \mathrm{mg} \mathrm{1x/day.} \mathrm{Subsequently,} \mathrm{a} \mathrm{Candida} \mathrm{infection} \mathrm{and} \mathrm{oral} \mathrm{lesions} \mathrm{were} \mathrm{treated} \mathrm{with} \mathrm{clotrimazole} \mathrm{troche,} \mathrm{fluconazole} 200 \mathrm{mg}$ 1x/day for 1 week, prednisolone $5 \mathrm{mg} \mathrm{1x/day} \mathrm{on} \mathrm{alternating} \mathrm{days,} \mathrm{and} \mathrm{chloroquine} 250 \mathrm{mg} 1 \mathrm{x} /$ day.

\section{CHALLENGE IN DIAGNOSIS AND} TREATMENT

In May 2017, her physician referred her to the Oral Medicine Clinic at the Faculty of Dentistry, Chulalongkorn University because her oral lesions showed no improvement.

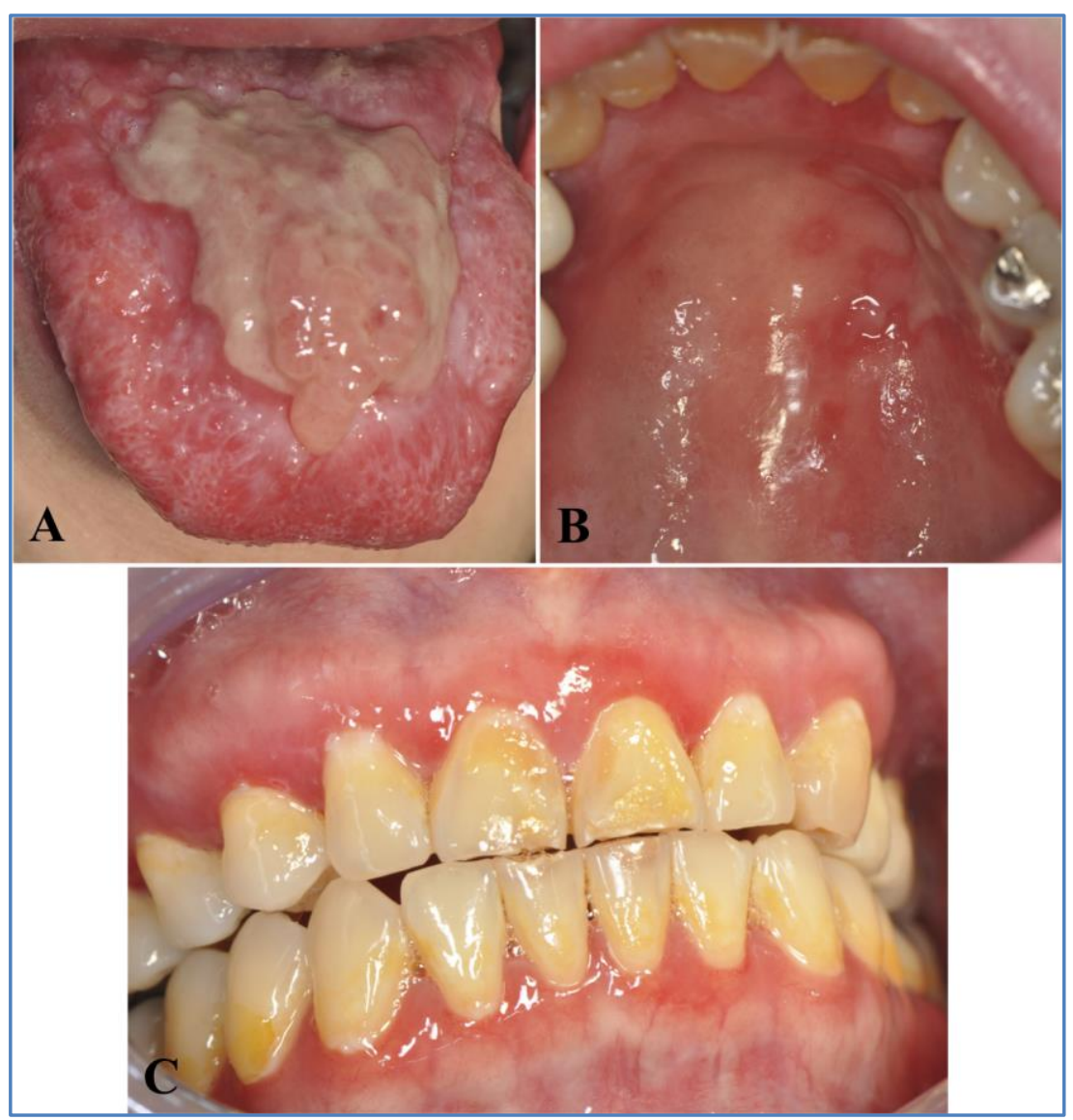

Examination revealed she had an exanthematous skin rash, a pseudomembrane-covered lobular mass on the tongue dorsum, palatal mucosa erosive lesions, and generalized erythematous gingivae (Figure 1A, B, and C). The complete blood count (CBC) showed neutropenia, lymphopenia, and was ANApositive with a titer of 1:320 (speckled pattern). A smearing from the oral lesion with $10 \% \mathrm{KOH}$ was positive for Candida. Moreover, many streptococci were present in the lesions. Mycoplasma pneumonaie and herpes simplex virus IgG assays were negative. Moreover, Gram-staining, AFB culture, Vincent culture, sensitivity test, red cell folate, serum folate, serum iron, total iron binding capacity, and serum vitamin $\mathrm{B}_{12}$ were all normal. Topical treatment with tetracycline mouthwash was prescribed to treat the oral lesions.

In June 2017, the lesions on the tongue, gingiva, and palate had slightly responded to treatment. Biopsy specimens were taken from the lateral border and dorsal surface of the tongue. The specimen from the lateral

Figure 1. A) Lobular mass with pseudomembranous cover on dorsum of the tongue; B) Erosive palatal lesions; C) Generalized erythematous gingiva at the first visit. 


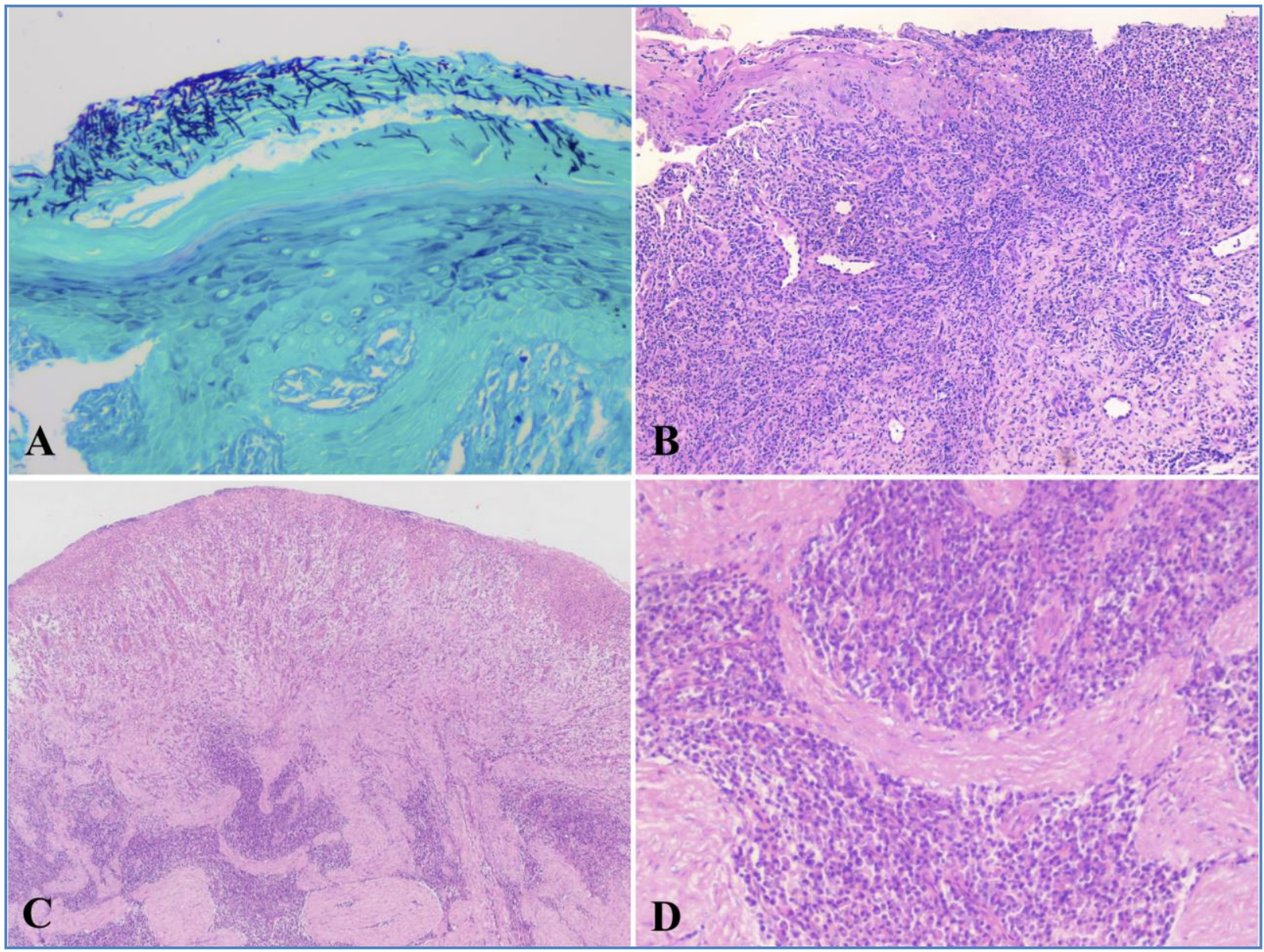

Figure 2. A) Periodic Acid Schiff staining showing positivity for Candida hyphae at the superficial part of the surface epithelium of the lateral border of the tongue (magnification X20); B) Hematoxylin and eosin (H\&E) staining of the lateral border of the tongue revealed the oral mucosa exhibiting loss of some part of the surface epithelium. The ulcerated area was covered by fibrinous exudate and the base of the ulcer was diffusely infiltrated by lymphocytes and plasma cells (magnification X10); C) Lower-power view of the H\&E staining revealing of the dorsum of the tongue which demonstrates an ulcerated area and inflammatory cells infiltrate in the underlying connective tissue (magnification X4); D) Higher-power view (magnification X20) of the H\&E staining displaying the chronic inflammatory cells infiltrate primarily consisting of dense plasma cells.

border of the tongue lesion was positive for PAS staining and demonstrated dense Candida hyphae at the superficial epithelium (Figure 2A) Hematoxylin and eosin (H\&E) staining of the lateral border of the tongue revealed the oral mucosa exhibiting loss of some part of the surface epithelium (Figure 2B).

$\mathrm{H} \& \mathrm{E}$ staining from the dorsum of the tongue reveals an oral mucosa exhibiting some surface epithelium loss. The ulcerated area was covered by a fibrinous exudate (Figure 2C). Higherpower view of the H\&E staining revealed numerous blood vessels and inflammatory cellular infiltrate were present in the connective tissue. Dense plasma cell infiltration was seen in the underlying connective tissue (Figure 2D). Moreover, chrome agar culture for Candida showed green pigment, consistent with Candida albicans.

From her history, clinical findings including oral manifestations, laboratory investigations, and histopathological report, plasma cell mucositis (PCM) was a possible diagnosis.
Therefore, hydrogen peroxide mouthwash $1 \%$ diluted with the water, Triamcinolone mouthwash $0.1 \%$ were applied to the generalized lesions at the first visit for 2 weeks. Subsequently, Fluocinolone acetonide $0.1 \%$ clotrimazole $1 \%$ gel was applied on the gingival lesions and clobetasol propionate $0.05 \%$ was applied on her buccal mucosae lesions. During the follow-up period, pseudomembranous candidiasis eruption lesions were treated with nystatin oral suspension $100,000 \mathrm{U} / \mathrm{ml}$ and the patient was advised to stop topical steroids. In July 2017, the lobular mass on the tongue dorsum was reduced in size. A soft splint was used to hold fluocinolone acetonide $0.1 \%$ clotrimazole $1 \%$ gel on the palate. At follow-up, in September 2017, she had developed dry eyes/mouth and a Schirmer tear test result of $2 \mathrm{~mm}$ of moisture on the filter paper in $5 \mathrm{~min}$. Therefore, dry eyes with possible Sjogren's syndrome was diagnosed; her ophthalmologist treated her with chloroquine and dry eyes were treated with artificial tear eyedrops. 


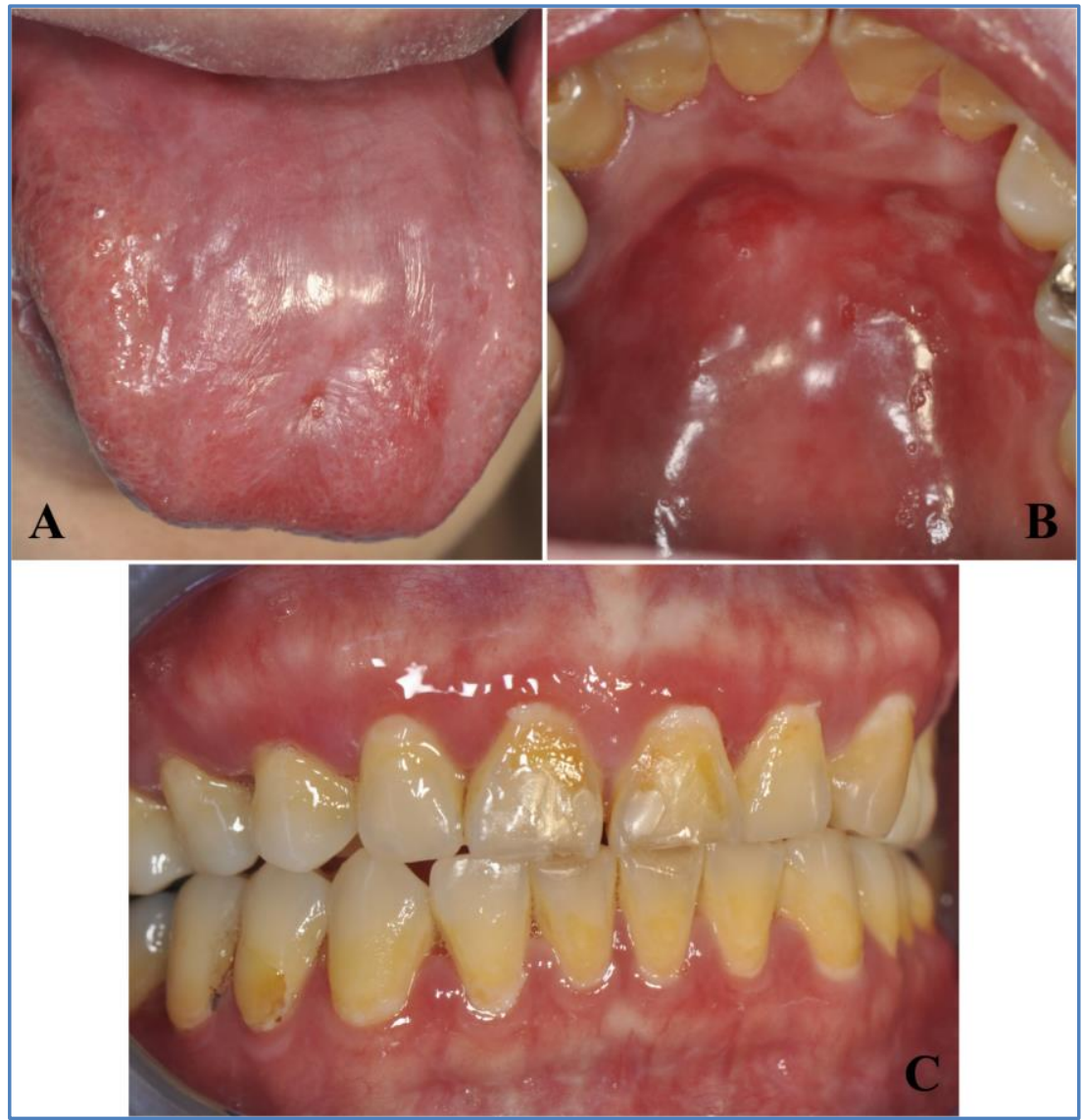

Figure 3. A) Dorsal of the tongue lesion showed improvement; B) Palatal lesions showed mild improvement; C) Generalized gingiva showed mild erythematous area.

for diagnosis and differential diagnosis from other lesions (Khan et al. 1977; Timms \& Sloan, 1991; Ferreiro et al., 1994).

Moreover, severe mucosal involvement and mild skin rash induced from Mycoplasma pneumoniae infection, called Mycoplasma induced-rash and mucositis (MIRM), is also uncommon (Poddighe \& Bruni, 2017). MIRM is characterized by non-specific skin rashes and remarkable mucosal lesions; these often involve the oral cavity and are very painful, steroid therapy in addition to macrolides may shorten the clinical course (Pereyre et al., 2016).

However, this case might possibly involve a hypersensitivity reaction to bacterial- M. pneumoniae, as there were many reactive plasma cells in the tongue biopsy specimen connective tissue. Typically, when bacteria or vi-

Although plasma cell mucositis often involves the oral and genital mucosa, there was no genital involvement in this case. Her tongue lesion was markedly reduced in size $(12 \times 2 \mathrm{~mm})$. Her pain score was lower, and her quality of life was improved.

The lobulated tongue lesion gradually reduced since July 2017, and completely disappeared in December 2017. Surprisingly, the gingiva showed mild erythematous areas, however, the palatal lesions were refractory to treatment (Figure 3A, B, and C). Her VAS for pain (5 at the first visit) was 0 . We recommended her to treat her gingival/palatal lesions with low intensity laser therapy. The timeline of the development of oral lesions and treatment are shown in Figure 4.

\section{DISCUSSION}

PCM of the oral cavity is a rare disease whose treatment and management is difficult. PCM is characterized by a sore mouth, intensely erythematous gingiva, a lobulated mass on the dorsal surface of the tongue, and oral mucosal lesions (Regezi et al.1972; Khan et al. 1977; Timms \& Sloan, 1991; Ferreiro et al., 1994; Smith et al., 1999). The histopathological findings from the dorsal tongue lesion in PCM showed a dense plasma cell infiltrate in the connective tissue. Therefore, appropriate investigations for severe oral mucosal involvement are important ruses enter the body, some B cells develop into plasma cells. The plasma cells make antibodies to fight bacteria and viruses and stop infection and disease (Nutt et al., 2015).

MIRM, a syndrome distinct from Stevens-Johnson syndrome and erythema multiforme, is a possible diagnosis in PCM (Canavan et al., 2015). To our knowledge, there is no publication linking MIRM and PCM. A review stated that synchronous autoimmune disease or immunologically mediated disease is associated with most PCM cases, including Sjogren's syndrome (Solomon et al, 2008). Taken together, the clinical presentation, histopathological findings, and autoimmune disease involvement such as developing Sjogren's syndrome in this case supported the diagnosis of PCM.

We found in this recalcitrant PCM case that potent topical steroid, Fluocinolone acetonide in solution (FAS) $0.1 \%$, treatment was effective on the lobulated tongue lesion. Various treatments have been used to treat PCM, including prednisolone, isotretinoin, intralesional triamcinolone acetonide injection, dapsone, cyclosporine, low dose radiation, $\mathrm{CO}_{2}$ laser, topical antifungal, levamisole, or resection (Solomon et al., 2008). However, marginal improvement, relapse, no recurrence, or remission during the follow-up were reported.

To our knowledge, this is the first case of PCM that described the lobulated lesion on the dorsal surface of the tongue responded well to topical FAS $0.1 \%$. The mass on the tongue 


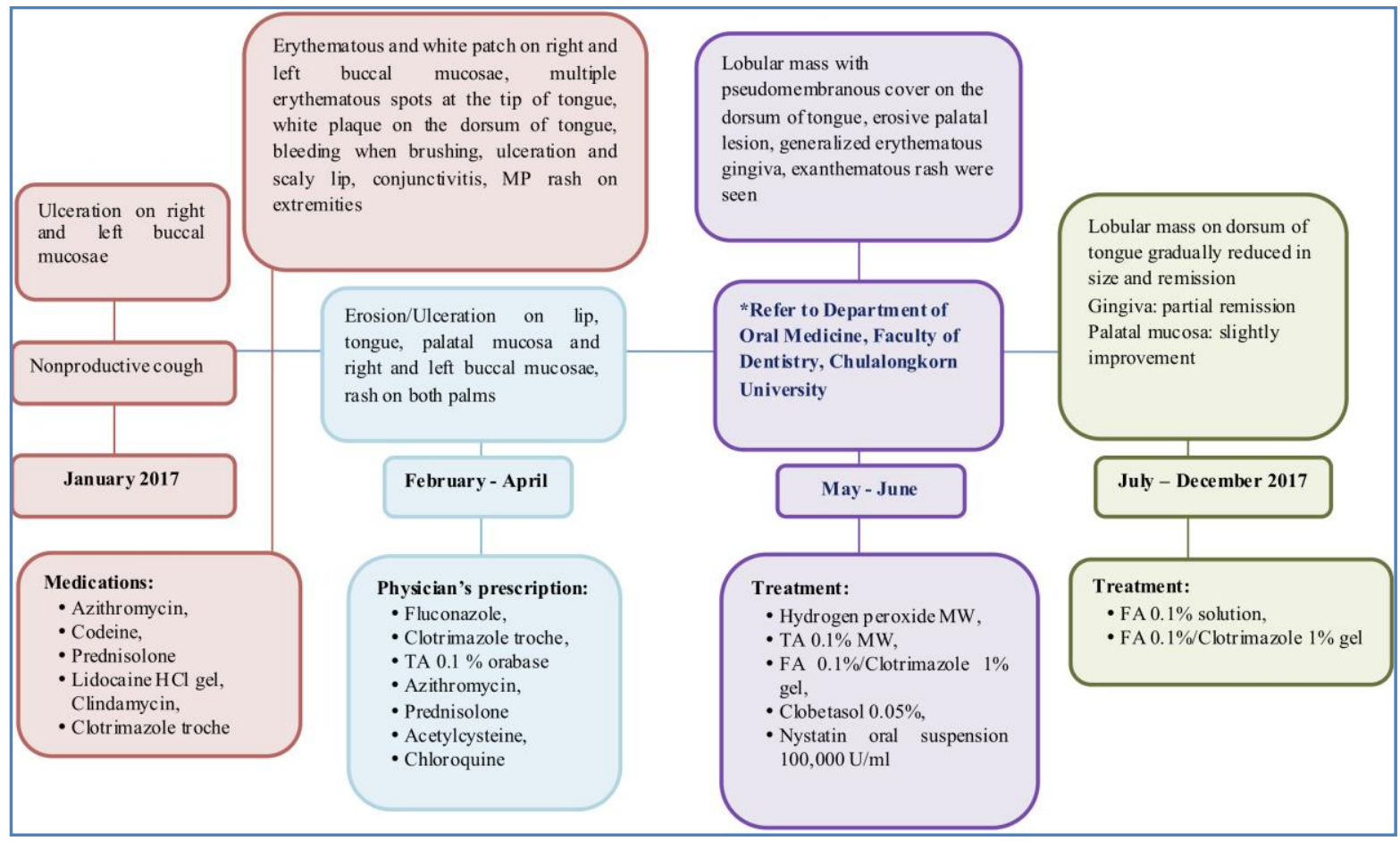

Figure 4. Timeline of oral lesions and treatment

gradually reduced in size until complete remission after surface with atrophic glossitis that might be related to the treatment for 7 months. Unfortunately, her tongue showed a smooth extensive use of ultra-potent topical steroids. Treatment and management of PCM mainly focuses on relieving symptoms. Her tongue lesions showed improvement, perhaps because the topical FAS $0.1 \%$ acted directly on the inflammatory cells in the lesion. However, although the gingiva showed partial improvement, the palatal lesions only slightly improved.

This patient was satisfied with the results of our treatment, only slight symptoms remained, and her quality of life was improved.

\section{Acknowledgements}

We express our sincere thanks to the physicians at Siriraj Hospital Bangkok, Thailand for providing the investigations, information, and previous treatment of this case. We thank the Research Unit in Oral Diseases and Oral Medicine staff for their assistance. We also thank Assist. Prof. Dr. Ekarat Phattarataratip for his kind assistance on this manuscript. Our thanks to Ms. Wanpen Sinheng, Microbiology Department, Faculty of Dentistry, Chulalongkorn University for her kind laboratory investigations, and Dr. Kevin Tompkins for manuscript and English editing. Thanks Dr. Sarinthorn Pariyawathee for helping edit the figures. We also thank Mr. Somchai Yodsanga, Oral
Pathology Department, Faculty of Dentistry, Chulalongkorn University for taking histopathological images

\section{Funding Source}

None

\section{Conflict of Interest}

None

\section{References}

Canavan, T.N., Mathes, E.F., Frieden, I., Shinkai, K. (2015).

Mycoplasma pneumoniae-induced rash and mucositis as a syndrome distinct from Stevens-Johnson syndrome and erythema multiforme: a systematic review. J Am Acad Dermatol, 72 (2): 239-45.

Ferreiro, J.A., Egorshin, E.V., Olsen, K.D., Banks, P.M., Weiland, L.H. (1994). Mucous membrane plasmacytosis of the upper aerodigestive tract. Am J Surg Pathol, 18 (10): 1048-53.

Khan, N.A., McKerrow, W.S., Palmer, T.J. (1997). Mucous membrane plasmacytosis of the upper aerodigestive tract. A case report with effective treatment. J Laryngol Otol, 111 (3): 293-5.

Nutt, S.L., Hodgkin, P.D., Tarlinton, D.M., Corcoran, L.M. (2015). The generation of antibody-secreting plasma cells. Nat Rev Immunol, 15 (3): 160-71. 
Pereyre, S., Goret, J., Bébéar, C. (2016). Mycoplasma pneumoniae: Current Knowledge on Macrolide Resistance and Treatment. Front Microbiol, 7: 974.

Poddighe, D., \& Bruni, P. (2017). Mycoplasma pneumoniaeinduced rash and mucositis (MIRM): an unusual mild skin rash associated with severe mucosal involvement. BMJ Case Rep, pii: bcr-2017-220749

Regezi, J.A., Taylor, C.G., Spinelli, F.R. Jr., Lucas, R.N., Travis, A.F.B. (1972). Allergic gingivostomatitis with laryngeal manifestations; report of a case. J Oral Surg, 30 (5): 373-7.
Smith, M.E., Crighton, A.J., Chisholm, D.M., Mountain, R.E. (1999). Plasma cell mucositis: a review and case report. J Oral Pathol Med 1999, 28 (4): 183-6.

Solomon, L.W., Wein, R.O., Rosenwald, I., Laver, N. (2008). Plasma cell mucositis of the oral cavity: report of a case and review of the literature. Oral Surg Oral Med Oral Pathol Oral Radiol Endod, 106 (6): 853-60.

Timms, M.S., \& Sloan, P. (1991). Association of supraglottic and gingival idiopathic plasmacytosis. Oral Surg Oral Med Oral Pathol, 71 (4): 451-3. 